\title{
Liver Transplantation for Alcoholic Liver Disease: Absence of Evidence for the Relevance of Abstinence
}

\author{
James O’Beirne $^{1}$ \\ Published online: 3 April 2020 \\ ○) Springer Science+Business Media, LLC, part of Springer Nature 2020
}

Liver failure due to alcohol-related liver disease (ArLD) has become a globally accepted indication for liver transplantation (LT). Despite the excellent reported outcomes, LT for this indication is often controversial and traditionally only available to select a group of patients who are considered to be ideal candidates based primarily on the outcome of enforced 6-month abstinence. The "6-month rule" had been widely adopted prior to LT in order to allow time for potential liver recovery and to predict post-LT abstinence. The rule can be challenged on two fronts. Firstly, many of the sickest patients with ArLD will simply not have the opportunity to complete this interval without succumbing to complications of the disease. Secondly, while the length of abstinence is a factor important for predicting future abstinence, the level of evidence is insufficiently robust and potentially could lead to denial of LT to otherwise good candidates.

These issues have been recently brought into sharp focus by the recent interest in LT for patients with severe acute alcoholic hepatitis (AH). In this issue of DDS, two excellent reviews provide the reader with a state-of-the-art update on the key questions in the field of LT for ArLD.

Mellinger and Stine [1] reviewed the growing body of published data for the efficacy of LT for AH. They show that the overall outcomes of LT for AH are excellent and similar to those reported for LT for other indications. Encouragingly, the rates of relapse to alcohol use (recidivism) appear to be similar to LT for conventional ArLD. Importantly, Mellinger and Stine remind us that there is much work to do toward improving outcomes for patients undergoing LT for this emerging indication with a need for standardization of the definitions of $\mathrm{AH}$ and non-response to treatment, and importantly prediction and management of relapse. The authors acknowledge that while relapse rates are not excessively high

James O'Beirne

James.OBeirne@health.qld.gov.au

1 University of the Sunshine Coast, Sippy Downs, QLD,

Australia in the current literature, since relapse is associated with graft dysfunction and failure, ongoing research is needed to better understand the prediction of relapse and its management on order to avoid the adverse outcomes in terms of graft survival and ethical, public, and professional confidence in LT for AH.

This theme is further developed in the comprehensive review by Professor Neuberger [2] on the risks of relapse. This review discusses the currently available tools to predict relapse and the limitations associated with their use. The impact of alcohol use on post-LT outcomes is highlighted (including in those transplanted for other indications) as is the need for continuous assessment for alcohol use postLT in order to identify patients who may benefit from early interventions. Professor Neuberger makes the important point that relapse to alcohol use is not the only concern in the management of ArLD patients undergoing LT given the increased risk of aerodigestive cancers in this group.

Both reviews are timely and are broadly supportive of increased access to LT for patients with ArLD, especially $\mathrm{AH}$. The need for more research on the prediction and management of relapse is highlighted by both articles; surely this will be facilitated by a structured approach to this important and emerging indication.

\section{References}

1. Mellinger JL, Stine JG. Early liver transplantation for severe alcoholic hepatitis. Dig Dis Sci. (Epub ahead of print). https://doi. org/10.1007/s10620-020-06159-9.

2. Neuberger J. Liver transplantation for alcoholic liver disease: what is the risk and consequence of relapse? Dig Dis Sci. (Epub ahead of print). https://doi.org/10.1007/s10620-020-06127-3.

Publisher's Note Springer Nature remains neutral with regard to jurisdictional claims in published maps and institutional affiliations. 